\title{
Fuzzy Numbers Applied in Reliability Assessment of Unreinforced Masonry Shear Wall
}

\author{
Mahdi Montazerolghaem ${ }^{1} \&$ Wolfram Jäger ${ }^{2}$ \\ ${ }^{1}$ University of Isfahan, Faculty of Engineering, Isfahan, Iran \\ ${ }^{2}$ Technical university of Dresden, Faculty of Architecture, Chair of structural design, Dresden, Germany \\ Correspondence: Mahdi Montazerolghaem, University of Isfahan, Faculty of Engineering, Isfahan, Iran. Tel: \\ 9891-3315-4228. E-mail: montazer9@gmail.com
}

Received: March 10, 2016

doi:10.5539/mas.v10n6p147
Accepted: March 27, 2016

Online Published: April 10, 2016

URL: http://dx.doi.org/10.5539/mas.v10n6p147

\begin{abstract}
In order to have safe and economy construction, different sources of uncertainty should be properly characterized and considered in structural design and verification. A reliability analysis is run to assess the consistency of design process, including the uncertainty. A full probabilistic approach is an appropriate means in considering the aleatory portion of uncertainty. In dealing with epistemic uncertainty in reliability analysis, modern mathematical tools like fuzzy logic is required. The non-deterministic design in a case study on Unreinforced Masonry shear Wall (URMW) by applying fuzzy numbers has performed. Instead of uncertain deterministic data of material strength, a range of possible numbers in the form of fuzzy numbers introduced to the model, considering the experiences and the expert knowledge. The predicted capacity which is fuzzy number provide more insight into behavior of URMW. Moreover, the study on significant influence of each variable on the ultimate capacity of URMW is easier. Several reliability analysis are run using only stochastic method with using fuzzy numbers. The effect of model uncertainty on assessed reliability is highlighted. The distinction between linear and non-linear application of partial safety factors is assessed. The result illustrate the fluctuation of reliability level of URMW for a wide range of applied normal force and different materials.
\end{abstract}

Keywords: fuzzy numbers, reliability, uncertainty, unreinforced masonry, shear wall

\section{Introduction}

The main function of structural Unreinforced Masonry Wall (URMW) is to bear the axial load. The behaviour of URMW subject to a pure axial force (centric load) is the matter of many scientific assessments. However, in real boundary condition, the interconnection of other structural elements (such as effect of floor slab on wall) or external horizontal forces (such as wind load) may cause some in-plane or out-of-plane eccentricity in URMW.

Traditionally, researchers may relied on the tools provided by probability theory to deal with problems in which uncertainty plays a substantive role. In recent years, it has become clear that dealing with uncertain models and imprecise data and running analysis based on a pure probability method is not adequate. The epistemic uncertainty does not have statistical nature, accordingly probabilistic methods are not appropriate in assessing the reliability level.

Moreover, the structural reliability encounters with very low level failure probability (e.g. $P_{f} \approx 10^{-5}$ ). This makes the analysis of reliability very sensitive to input data, especially when the extreme value distributions are of concern. In this regard, the contribution of uncertainty model becomes very crucial. For instance, a small number of sample elements (as it happens usually in structural experiments) can lead to significant approximation (inaccuracy) in statistical parameters and therefore inaccurate results of probabilistic analysis.

Nevertheless, the theory of fuzzy sets introduced by L. A. Zadeh (1965) makes experiences and expert knowledge accessible for controlling undesired scatter nature or lack of data. Combination of stochastic modelling and fuzzy set provides even more powerful bases for reliability analysis. the non-precise data in form of fuzzy numbers were first addressed in the 1980ies and the first articles combining fuzzy imprecision and stochastic uncertainty were run by see Lotfi A. Zadeh (1984) and Kacprzyk and Fedrizzi (1988).

The purpose of this study is twofold: to apply Fuzzy numbers in URMW design and to insert fuzzy numbers in Randomness method in order to assess the reliability of URMW subject to in-plane shear load designed 
according to Eurocode 6 (DIN, 2012).

\section{Uncertain Data}

Due to fluctuating nature of surrounding conditions, all available quantities of material properties recorded from experiments are imprecise. With similar reasons, all the forecast values in predicting load parameters are inaccurate. The prediction in both load combination and resistance models may be even more uncertain, due to additional approximation involved in the idealized model, which is used to simulate or describe the real sense of the matter. This phenomenon is named uncertainty and is discussed in different fields of science.

In general, uncertainty can be of two epistemic and aleatoric uncertainty categorised. Epistemic uncertainty is objective and is related to the insufficient data or lake of knowledge. The epistemic uncertainty may be decreased by raising the quality and quantity of observations. In contrast, aleatory uncertainty is subjective and is related to random and stochastic behaviour of individual element when the same inputs yield scattered outcome in the same system.

The common categorization of uncertainty is applied to conclude that the aleatory part of uncertainty could be evaluated by the probability theory since it satisfies the statistical laws, and possesses purely objective information, and it might be referred to as "randomness".

The idea that statistical variation in a parameter could be considered in quantification of uncertainty and the specifying design values are not new and many standards have recognized this for some time. Within the probabilistic approach to structural safety and performance specification, a basic variable, X, may be characterized by two quantities, an "average" value and a "scatter measure". The former corresponds to an expected value or mean, the latter, to a distribution and coefficient of Variation, Cov.. As it will be observed, probabilistic approach provides appropriate platform to implement aleatoric uncertainty.

On the contrary, "fuzziness" results from epistemic uncertainty, which is not characterized by random properties but subjective influences and is dealt with on the bases of fuzzy set theory. Fuzziness possesses subjective and objective information.

In logical sense, uncertainty measurement intends to work towards reducing epistemic uncertainties to aleatoric uncertainties. Kiureghian and Ditlevsen (2009) caegorize uncertainties within a model since it then becomes clear which uncertainties have the potential of being reduced.

In most applications, engineers confront with both randomness and fuzziness. Because of incompleteness and imprecision, the observed uncertainty does not fulfil all preconditions of stochastic uncertainty, but instead it exhibits partial stochastic properties. More information and examples could be found in Möller and Beer (2004) and Cai (1996). Depending on application, there exist other techniques and tools to estimate and maintain uncertainty and provide an acceptable accuracy in the outcome like Karhunen-Loève's, polynomial chaos expansions and evidence theory, Blake (1966).

\subsection{Interval Data}

Many measurements (e.g. material strength) are connected with numerous amount of uncertainties. An example is the working life time of a structure that in general may not be described by one real number because the end of the life time is not a precise number $\left(x_{0} \in \mathbb{R}\right)$ but more or less non-precise. This kind of non-precise data may easily be described by interval arithmetic.

The intervals $([a, b] \subseteq \mathbb{R})$ are uniquely characterized by the indicator functions, $I_{[a, b]}$. The indicator function $I$ of a classical set $A$ is defined by:

$$
I_{A}(\mathrm{x})= \begin{cases}1 & \text { for } x \in A \\ 0 & \text { for } x \notin A\end{cases}
$$

Viertl in (1996) and in (1997) developed an approach to describe the non-precise observations named non-precise numbers, in a mathematical manner where $x^{*}$ are modelled by characterizing functions $\xi(x)$, which characterize the imprecision of an observation. A characterizing function $\xi(x)$ is a real function of a real variable with the following properties:

(1) $\xi: \mathbb{R} \rightarrow[0,1]$

(2) $\exists x_{0} \in \mathbb{R}: \xi\left(x_{0}\right)=1$

(3) $\forall x_{0} \in(0,1]$ the set $B_{\alpha}:=x \in \mathbb{R}: \xi(x) \geq \alpha=\left[a_{\alpha}, b_{\alpha}\right]$ is a finite closed interval, named $\alpha$-cut $(\alpha$-level) of the $\xi(x)$ 
Interval mathematics and convex modelling offer additional modelling techniques, although these only allow for a binary assessment of the membership of elements in a set. In this content, the rough sets theory is contributively consider the existing zone between membership and non-membership. Fuzzy set theory in addition to rough sets theory allows the assessment of elements of a set based on a normalized scale ranging between zero and unity.

\subsection{Fuzzy Sets and Fuzzy Numbers}

According to B. Möller, W. Graf, and M. Beer (2000) the theoretical basis of fuzzy set was first introduced by L. A. Zadeh (1965) and later was extended by Bandemer and Gottwald (1989). Here a quick review on fuzzy set is presented by Chachi and Taheri (2011).

Let $X$ be a universal (convex, normalized) set, then the fuzzy set (or uncertain set), $\tilde{\mathrm{A}}$ of $X$ is defined by its membership function $\tilde{\mathrm{A}}: X \rightarrow[0,1]$. The membership function is at least segmentally continuous. From the uncertain set $\tilde{\mathrm{A}}$, the crisp sets are derived.

$$
\tilde{\mathrm{A}}_{\alpha k}=\left\langle x \in X \mid \tilde{\mathrm{A}}(x) \geqq \alpha_{k}\right\rangle
$$

The set is named the $\alpha$-level set of $\tilde{\mathrm{A}}$ for $\alpha_{k} \in(0,1]$, and $\tilde{\mathrm{A}}_{0}$ is the closure of the set $\langle x \in X| \tilde{\mathrm{A}}(x)>$ 0 ). It is assumed that: 1) $X$ is endowed with a topological structure; and 2) the universal set $X$ is a set of real numbers (i.e. $X=\mathbb{R}$ ). The fuzzy set $\tilde{\mathrm{A}}$ is called a fuzzy number, if each $\tilde{\mathrm{A}}_{\alpha}$ is a nonempty closed interval for all $\alpha \in(0,1]$, it will be presented by $F(\mathbb{R})$. The set of all fuzzy numbers are associated with $\mathbb{R}$. In fuzzy set theory, the characterizing function is named membership function. A common kind of fuzzy number is the triangular fuzzy number with a linear membership function, which is denoted by triplet number, $\tilde{A}=\left\langle a_{l}, a, a_{r}\right\rangle$, where $a_{l}, a, a_{r}$ are the left, the centre and the right spread values of $\tilde{\mathrm{A}}$, respectively; the centre, $a$, which can be referred to as the mean value has unit membership, see Fig. 1-a.

The membership function and the $\alpha$-level set of a triangular fuzzy number $\tilde{\mathrm{A}}$ are defined in Eqs. (3), where, $I($.$) is the indicator function. \tilde{\mathrm{A}}$ is named as fuzzy point (crisp number) with value $m$, provided that membership function of the set is $\tilde{\mathrm{A}}(x)=I_{m}(x)$. It is easy to observe that, $\tilde{\mathrm{A}}_{\alpha}=m$ for all $\alpha \in[0,1]$. The mathematical expression of the above is presented in Eqs. (3)

$$
\begin{aligned}
& \tilde{A}(x)=\frac{x-\left(a-a_{l}\right)}{a_{l}} I_{\left[a-a_{l}, a\right]}(x)+\frac{\left(a-a_{r}\right)-x}{a_{r}} I_{\left[a, a+a_{r}\right]}(x) \\
& \tilde{A}_{\alpha}=\left[a_{\alpha}^{l}, a_{\alpha}^{r}\right]=\left[a-(1-\alpha) \cdot a_{l}, a+(1-\alpha) \cdot a_{r}\right]
\end{aligned}
$$

A general membership function, which can be any continuous convex function meets the conditions (1) and (2) of intervals is shown in Fig. 1-b. There the non-precise interval numbers are special fuzzy subsets of $\mathbb{R}$, in another word, the fuzzy subsets are generalization of intervals. In every convex uncertain set $\tilde{\mathrm{A}}$, each $\alpha-$ level set $A_{\alpha k}$ is an interval $\left[x_{a_{k} l}, x_{a_{k} r}\right]$ where,

$$
\begin{aligned}
x_{a_{k} l} & =\min \left\langle x \in X \mid \tilde{\mathrm{A}}(x) \geq \alpha_{k}\right\rangle \\
x_{a_{k} r} & =\max \left\langle x \in X \mid \tilde{\mathrm{A}}(x) \geq \alpha_{k}\right\rangle
\end{aligned}
$$

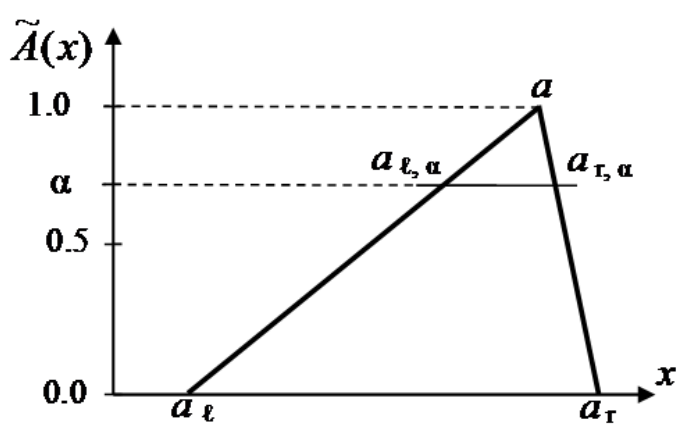

a)

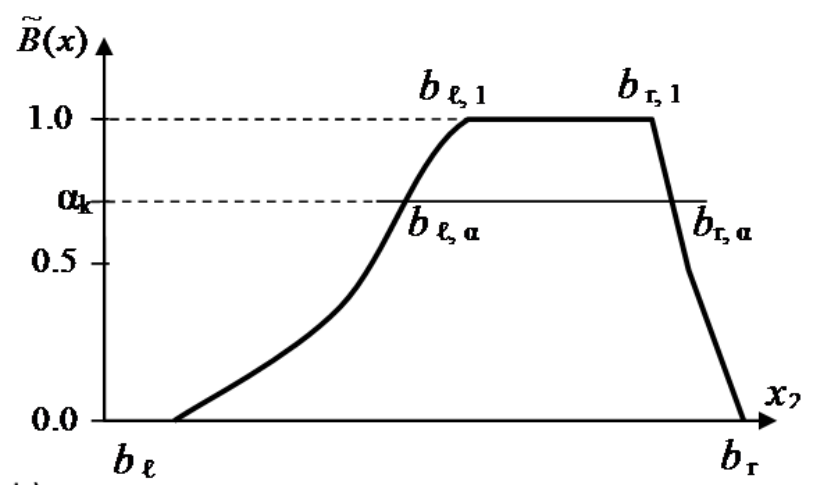

b)

Figure 1. a) membership function of triangular fuzzy number; b) general convex membership function of fuzzy number 
It should be mentioned that in static and dynamic application the non-convex fuzzy variables and non-convex fuzzy random variables might appear. Reuter (2009) improved the analysis methods and specification of fuzzy numbers to allow the modelling of uncertain data as non-convex fuzzy variables and non-convex fuzzy random variables. This was achieved by developing a generalized discretization algorithm by extending the simulation methods of convex fuzzy random variables.

Möller and Beer (2004) extended non-deterministic methods in order to account for non-stochastic uncertainty. The epistemic uncertainty of a parameter is characterized and implemented into analysis by means of the "membership function". Therefore defining membership function is a key step in calculation. Membership functions for structural parameters may be specified based on experiments. In addition to the measured values, it is necessary to consider the extent of samplings, possible errors in measurements and other inaccuracies, estimates made by experts, experience gained from comparable problems and so on.

Based on fuzzy set theory, B. Möller et al. (2000) developed and formulated a general method for fuzzy structural analysis in terms of $\alpha$-level optimization with the application of a modified evolution strategy. The fuzzy verification is a sort of deterministic analysis. The objective of this approach is to supplement probabilistic methods in a means that uncertainties in their natural form (characteristics) may be accounted for more appropriately.

According to the subject under study, the designer specifies the membership function, $\mu_{A}(x)$, for each parameter or uncertain variable. Then, the realistic simulation of a structure with the aid of a crisp (or uncertain) algorithm applied to fuzzy values for input and model parameters is performed. Several algorithms for static and dynamic fuzzy structural analysis are adopted as a deterministic fundamental solutions.

By the means of this realistic simulation algorithm the complex loading processes are treated in an incremental-iterative manner with respect to all essential nonlinearities. The fuzzy analysis algorithm maps $n$ fuzzy input values $\tilde{x}_{i}$ and $p$ fuzzy model values, $\widetilde{m}_{r}$, into $\tilde{\mathrm{Z}}_{\mathrm{j}}$ result values, which are fuzzy values as well, by means of the extension principle in combination with the Cartesian product between fuzzy sets by L. A. Zadeh (1965) and B. Möller et al. (2000). However, the extension principle is hardly practicable in the case of complex mapping operators, as its application requires discretization of the support of the uncertain input set (e.g. using a point mesh). This leads to numerical problems. In order to develop an appropriate method for processing fuzzy input values and fuzzy model parameters the concept of $\alpha$-optimization is adopted by B. Möller et al. (2000).

\section{$2.3 \alpha$-Level Optimization of Fuzzy Values}

Fuzzy values may be discretized with the aid of $\alpha$-level sets. The $\alpha$-level sets $A_{i}, \alpha_{k}, i=1, \ldots, n$ of the fuzzy input values $\tilde{A}_{1}, \ldots, \tilde{A}_{i}, \ldots, \tilde{A}_{n}$ form the $\mathrm{n}$-dimensional crisp subspace $\underline{X}_{\alpha_{k}}$, of the x-space. For $\alpha_{k}=0$ the crisp support subspace is obtained.

If the fuzzy input values are convex uncertain sets and among them no interaction exists, an n-dimensional hyper-cuboid is obtained. The non-convex fuzzy input values lead to a disjoint subspace $\underline{X}_{\alpha_{k}}$. If there is interaction, the shape of the subspace $\underline{X}_{\alpha_{k}}$ generally departs from the shape of the n-dimensional hyper-cuboid.

All fuzzy input values are discretized through the same number of $\alpha_{\mathrm{k}}$-levels with $k=1, \ldots, r$. For each fuzzy input value $\tilde{x}_{i}=\tilde{A}_{i}$ on level $\alpha_{k}$ the $\alpha$-level set $A_{j, \alpha k}$ is then assigned to $\tilde{x}_{i}$ and the total of $A_{j, \alpha k}$ form the crisp subspace $\underline{X}_{\alpha_{k}}$. With the aid of the mapping operator $\underline{Z}=f\left(x_{1}, x_{2}, \ldots, x_{n}\right)$ it is possible to compute elements of the $\alpha$-level sets $B_{j, \alpha k}$ of the fuzzy result values $\tilde{z}_{j}=\tilde{B}_{j}, j=1, \ldots, m$ on the $\alpha$-level $\alpha_{k}$. The mapping of all elements of $\underline{X}_{\alpha_{k}}$ yields the crisp subspace $\underline{Z}_{\alpha_{k}}$ of the z-space.

Once the smallest element $z_{j, \alpha_{k l}}$ and the largest element $z_{j, \alpha_{k r}}$ of the $\alpha$-level set $B_{j, \alpha k}$ are found, the two points of the membership function $\mu\left(z_{j}\right)=\mu_{B_{j}\left(z_{j}\right)}$ become known. The search for smallest and largest elements may be formulated as an optimization problem. for this purpose the following objective functions must be satisfied:

$$
\begin{gathered}
z_{j}=f\left(x_{1}, x_{2}, \ldots, x_{n}\right) \Rightarrow \operatorname{Max} \mid\left(x_{1}, x_{2}, \ldots, x_{n}\right) \in \underline{X}_{\alpha k} \\
z_{j}=f\left(x_{1}, x_{2}, \ldots, x_{n}\right) \Rightarrow \operatorname{Min} \mid\left(x_{1}, x_{2}, \ldots, x_{n}\right) \in \underline{X}_{\alpha k}
\end{gathered}
$$

These equations are satisfied by the optimum points $\underline{x}_{\text {opt }}$. For each fuzzy result value, precisely two optimum points in the crisp subspace $\underline{X}_{\alpha k}$ belong to each $\alpha$-level $\alpha_{k}$. The optimization process for all $\alpha$-levels $\alpha_{k}$ and 
all fuzzy result values $\underline{z}_{j}$ is referred to as $\alpha$-level optimization.

In the optimization problem presented by B. Möller et al. (2000), for each of the $m$ fuzzy result values $\tilde{z} j$ on each one of the $r$-levels $\alpha_{k}$ must be solved twice, i.e. $(2 \cdot m \cdot r)$ times in total. A multiple solution for the optimization problem is necessary. The $\alpha$-level optimization thus demands a robust optimization technique which is independent of the type and behaviour of the objective function or restrictions and is capable of finding global optima in a realistic manner. Standard optimization methods are only partly appropriate for this purpose. For this reason a compromise solution is developed by combining the evolution strategy, the gradient method and the Monte-Carlo method.

The combination of directed and non-directed search techniques is found to be advantageous when compared to a purely directed search technique seeking for global optima. A mixed technique is less sensitive in relation to less "well-behaved" objective functions. By taking advantage of existing information concerning the behaviour of the objective function the number of "unnecessary" computations of objective function values (leading to lower results) is reduced. If the available information is insufficient, then the random-oriented methods are applied. Fuzzy numbers have been and are successfully used in steel and concrete structures' design as well as in monitoring or indicating damage of the buildings. Fuzzy analysis is capable for static loading processes and dynamic processes. Fuzzy analysis not only take into account the corresponding uncertainty in the inputs, but also provide additional information in output for the designer. For detailed information and illustrative examples refer to B. Möller et al. (2000), (2000) and (2001).

The fuzzy structural analysis promote the of load-bearing behaviour assessment subject to considered uncertainties. The " $\alpha$-level optimization" algorithm is quite a promising technique in this regards. Some attempts are made to apply fuzzy analysis as alternative to stochastic methods, Möller, Beer, and Liebscher (2005). Nevertheless, the aleatory uncertainty require the probabilistic tool to handle.

\section{Verification of URMW with Fuzzy Data}

There exist many basic theoretical approaches to predict the in-plane shear capacity of URMW. According to Kranzler (2008), the first studies on masonry shear strength were conducted by Kelch and Norman (1931) followed by, Benjamin and Williams (1958), Vogt (1961) and Zelger (1964) who focused on recalculation of experimental data and derived a shear strength $f_{v}$ directly from the test data. More recent studies in Germany consist of Mann and Müller (1978), Simon (2002) and Kranzler (2008).

Kranzler conducted massive FEM analyse and analytical calculations for various geometrical aspect ratio of wall by considering several unit size and masonry materials. Meanwhile, he integrated the available methods at the time and compared this outcome with the experimental results. He suggested that parameter $\psi$ accounts for the structural system of the wall. In general, a distinction may be drawn between the two boundary cases: for totally (full) restraint at top and the bottom of the wall, $\psi=0.5$ and for cantilever wall with no restraint at the top $\psi=1.0$.

Kranzler states that most of the theoretical models are derived from the equilibrium at a single unit in the panel centre and assume small units compared to the panel dimensions. In the middle of the panel, the assumption of a diagonal shifted stress blocks with similar state of stress for adjacent units is justified. However, at the bottom and the corner areas of a panel (where the calculation of the bearing capacity is mostly run), the resulting state of stress is not the same as the assumed one. Accordingly, he proposed additional criteria regarding wall boundary conditions and units' size.

The fuzzy input data for the basic variables of a case regarding Clay Brick wall (URMW CB1) designed by German National Annex of Eurocode 6 (DIN EN 1996-1-1/NA DIN EN 1996-1-1/NA (DIN, 2012)) are provided in Fig. 2. The analysis is run by $\alpha$-level discretization method in WinFuz (Note 1) software that is developed in the Technische Universität Dresden. The interval of normal force, $n_{E D}$, around each verification point is set in a manner to provide a continuous interval over $0<n_{E D}<0.7$ range. 

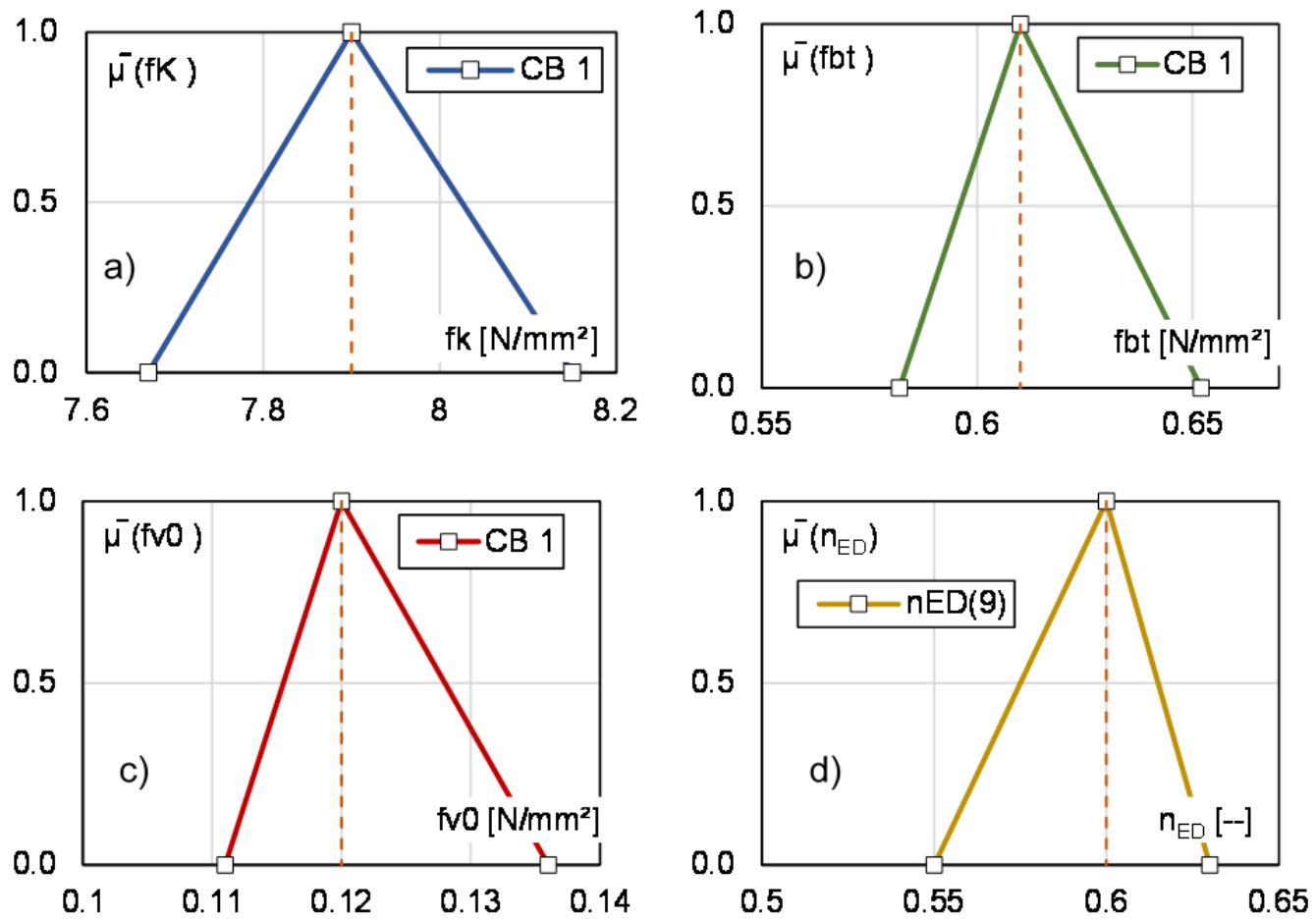

Figure 2. Fuzzy input data, (a) CB 1, masonry compressive strength, (b) CB 1, unit tensile strength, (c) CB 1, masonry initial shear strength, and (d) normal force of $\mathrm{i}=9, \tilde{n}_{E D}=\langle 0.55,0.6,0.63\rangle$

Figure 3 illustrates the result of fuzzy interval verifications for URMW CB 1 at several normal force intervals:
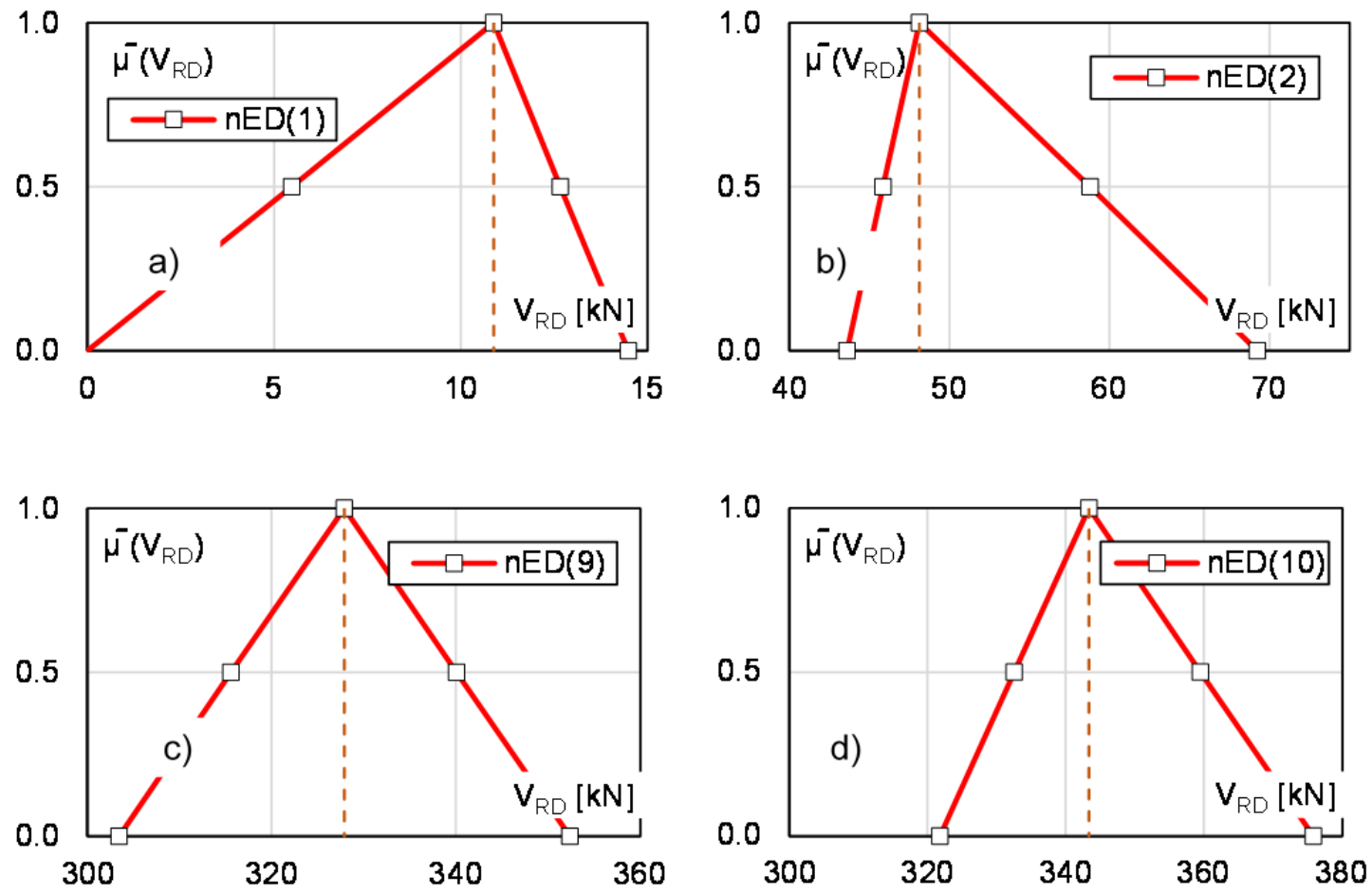

Figure 3. Fuzzy output data, of CB1 verification for several normal forces: (a) $\mathrm{i}=1, \tilde{\mathrm{n}}_{\mathrm{ED}}=\langle 0.0,0.005,0.0065\rangle$, (b) $\mathrm{i}=2, \tilde{\mathrm{n}}_{\mathrm{ED}}=\langle 0.0065,0.025,0.037\rangle,(\mathrm{c}) \mathrm{i}=9, \tilde{\mathrm{n}}_{\mathrm{ED}}=\langle 0.55,0.6,0.63\rangle$ and $\left.(\mathrm{d}) \mathrm{i}=10, \tilde{\mathrm{n}}_{\mathrm{ED}}=\langle 0.63,0.667,0.73\rangle\right)$ 
The result of fuzzy interval verifications over the entire normal force interval is illustrated in Fig. 4. The upper part of diagram (in blue) shows the behaviour of URMW when the partial safety factor, $\gamma_{M}$ is set to unity. The lower part (in red) indicate the design capacity of the wall where the partial safety factor of $\gamma_{M}=1.5$ is considered.

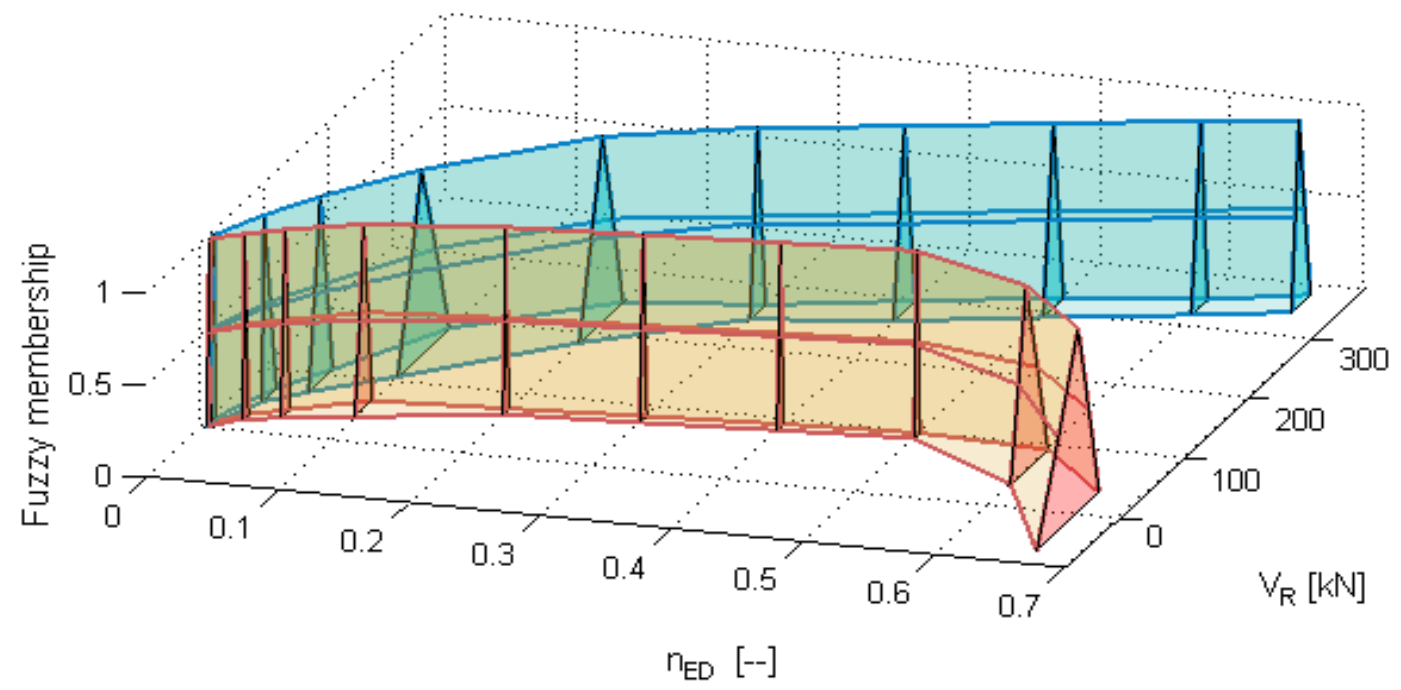

Figure 4. Verification of URMW (CB 1) with Fuzzy data

\section{Fuzzy Random Variables}

As mentioned before, the combining fuzzy imprecision and stochastic uncertainty is suggested and exercised by researchers. In fact, the first comprehensive uncertainty modelling was developed according to fuzzy random variables theory. Randomness and fuzziness are considered simultaneously and the real-valued random variables and fuzzy parameters are included as special cases. For numerical evaluation, a formulation is developed in Technische Universität Dresden based on $\alpha$-discretization according to B. Möller et al. (2000) which is applied for structural reliability analysis.

\section{Reliability with Fuzzy Data}

Applying the uncertain computational models containing fuzzy model parameters result in a fuzzy limit state surface, expressed as follows:

$$
\tilde{g}(\underline{x})=0 \equiv\langle(g(\underline{x})=0 ; \mu(g(\underline{x})=0)|\underline{x} \in \underline{X}\rangle
$$

Similar to deterministic limit state, the fuzzy limit state surface subdivides the space of the fuzzy probabilistic basic variables into a fuzzy survival region and a fuzzy failure region.

In order to compute the elements $g(\underline{x})=0$, it is necessary to discretize the fuzzy model parameters (i.e. selection of an $\alpha$-level and selection of elements from the $\alpha$-level sets). By this means, possible values of the fuzzy model parameters are defined. These values serve as input data to a (non-linear) analysis algorithm through which the crisp limit state surface $g(\underline{x})=0$ is computed. The respective analysis algorithm is referred to as the deterministic fundamental solution. The quality of the deterministic fundamental solution has a decisive influence on the safety assessment results; thus, the system behaviour of the structure has to be simulated in a realistic numeric manner. The assessment of the points $\underline{x}$ in the space of the fuzzy probabilistic basic variables regarding failure or survival are carried out through membership functions. The membership function of the fuzzy failure region is expressed as follows:

$$
\mu(g(\underline{x}) \leq 0)=\left\{\begin{array}{lr}
1 & \forall \underline{x} \mid g(\underline{x})_{\alpha=1} \leq 0 \\
\mu(g(\underline{x})=0 & \text { otherwise }
\end{array}\right.
$$

In this context, the fuzzy probability of failure could be expressed as:

$$
\tilde{P}_{f}=P(\tilde{R}-\tilde{S} \leq 0)
$$

Fuzzy reliability $\tilde{\beta}$ is a fuzzy number defined as:

$$
\tilde{\beta}=-\phi\left(\tilde{P}_{f}\right)
$$


where, $\Phi$ is standard normal probability distribution and $\tilde{P}_{f}$ is the fuzzy failure probability. As explained before, the output of a fuzzy analysis is a fuzzy number. However, in current codes of practice, only real numbers are given as the reliability measure targets. In order to interpret the fuzzy reliability index, $\tilde{\beta}$, Möller, Graf, and Beer (2003) considered the following distinction:

1. The verification according to Eqs. (15) is fulfilled, given that none of the elements of $\tilde{\beta}$ are smaller than the standard target.

$$
\beta_{\alpha=0, l} \geq \beta_{\text {Target }}
$$

2. The verification according to (15) is not fulfilled, given that all elements of $\tilde{\beta}$ are smaller than target:

$$
\beta_{\alpha=0, r}<\beta_{\text {Target }}
$$

3. The verification according to (15) is considered as "partially fulfilled", given that the target value is an element of $\tilde{\beta}$, like:

\subsection{Structural Target Reliability Index}

$$
\beta_{\text {Target }} \in \tilde{\beta} \wedge \beta_{\alpha=0, l} \neq \beta_{\text {Target }}
$$

Codes of practice encompass different target reliability values. The proposed target reliability index in JCSS (2011) for ultimate limit states is tabulated in Table 1.

Table 1. Tentative target reliability indexes, $\beta$ (and associated target failure rates), related to 50 years reference period and ultimate limit state JCSS (2011)

\begin{tabular}{lccc}
\hline & \multicolumn{3}{c}{ Reliability indexes, $\beta$, according to consequences of failure } \\
\cline { 2 - 4 } Relative cost of safety measure & minor & moderate & major \\
\hline A (large) & 1.7 & 2.0 & 2.6 \\
B (medium) & 2.6 & 3.2 & 3.5 \\
C (small) & 3.2 & 3.5 & 3.9 \\
\hline
\end{tabular}

Target values of reliability index in DIN EN 1990:2002 (DIN, 2011) for various design situations, and for reference periods of 1 year and 50 years, are tabulated in table 2 . The values of $\beta_{T}$ in tables correspond to levels of safety for reliability class RC2 (see DIN EN 1990:2002 (DIN, 2011) Annex B) structural members.

Table 2. Target reliability indexes, $\beta$, related to life time reference period and ultimate limit state DIN EN 1990:2002 for Class RC2 structural members which is associated with the second consequence classes (CC2 Medium) (DIN, 2011)

\begin{tabular}{lcc}
\hline & \multicolumn{2}{c}{ Reliability indexes, $\beta$, according to consequences of failure } \\
\cline { 2 - 3 } Relative cost of safety measure & small & some \\
\hline Ultimate & 4.7 & 3.8 \\
Fatigue & - & 1.5 to 3.8 \\
Serviceability (irreversible) & 2.9 & 1.5 \\
\hline
\end{tabular}

* depends on degree of inspectability, reparability and damage tolerance

It should be noted that the partial factors given in EN 1990 up to EN 1999 generally lead to a structure design with a $\beta$ value greater than 3.8 for a 50 years reference period. In this study $\beta=3.8$ is chosen as the target value.

Further reference in different consideration on the specific case of fuzzy structural reliability analysis includes: Beer, Tong, Mingqiang, and Ferson (2011), Montazerolghaem, Sickert, Graf, and Jäger (2011), Yubin, Zhong, and Guangyuan (1997), Cai (1996), Grosse (2007), Möller, Graf, Hoffmann, and Sickert (2007), Möller et al. (2003), Reuter (2009), Pannier, Waurick, Graf, and Kaliske (2013), Graf, Götz, and Kaliske (2014) and Möller, Graf, Sickert, and Reuter (2007).

The probability of failure and reliability level of URMW designed in accordance to DIN EN 1996-1-1/NA DIN EN 1996-1-1/NA (DIN, 2012) corresponding to the different exemplary walls, made of Clay Brick (CB), Calcium Silicate (CS) and Autoclaved Aerated Concrete (AAC) are assessed in this article. 
It should be noted that in this study lognormal distribution is adopted with respect to the uncertainty in resistance model. In General, in order to characterize uncertainty in probabilistic models, the observed (experimental) capacity is compared with predicted value and then the relevant uncertainty in model is derived.

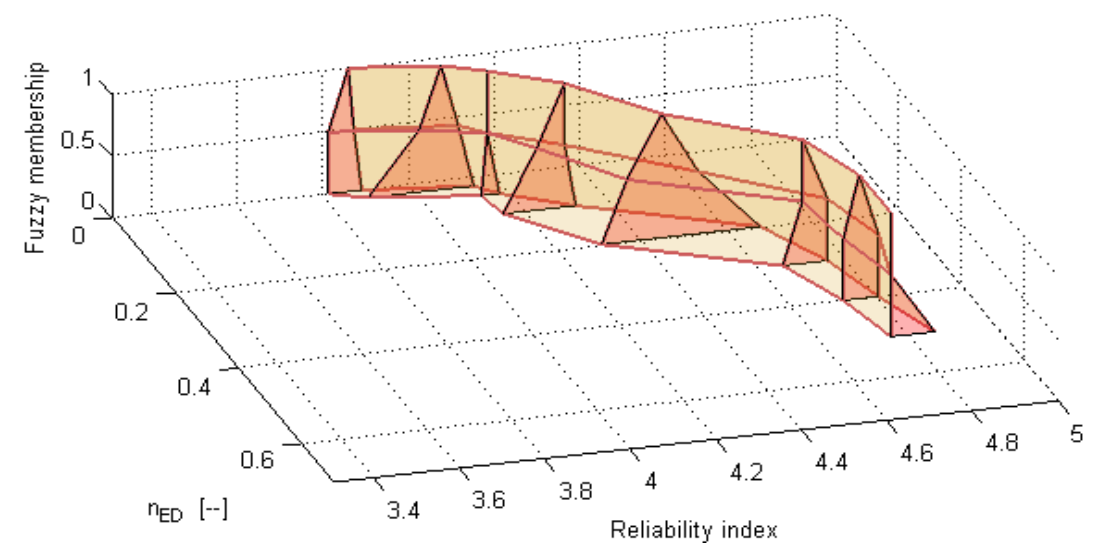

Figure 5. 3D-Reliability of CB URMW ( $\psi=1.0)$ through Fuzzy-Random data designed due to DIN EN1996-1-1/NA

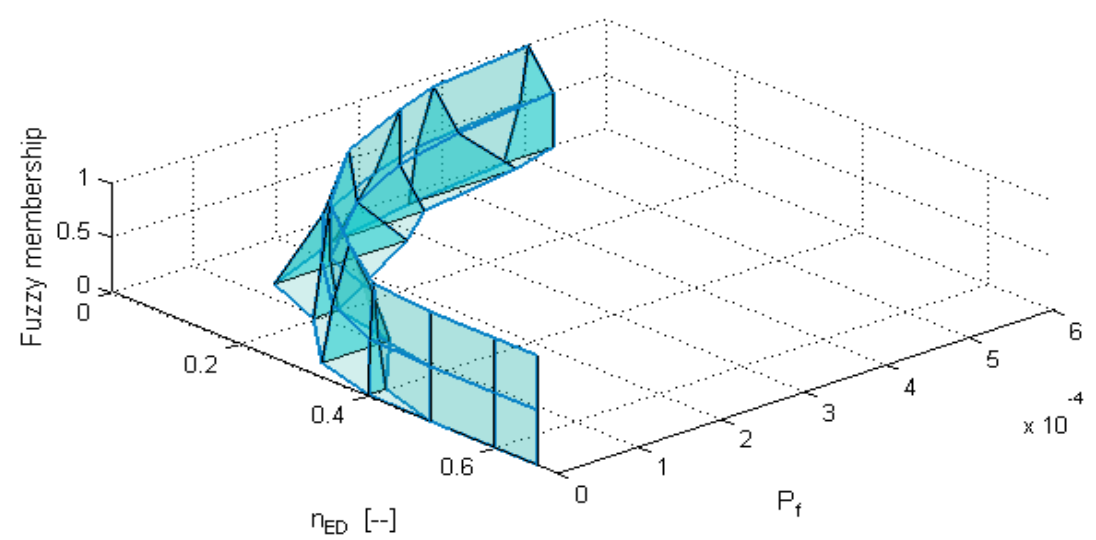

Figure 6. 3D-Failure probability of CB URMW $(\psi=0.5)$ through Fuzzy-Random data designed due to DIN EN1996-1-1/ NA

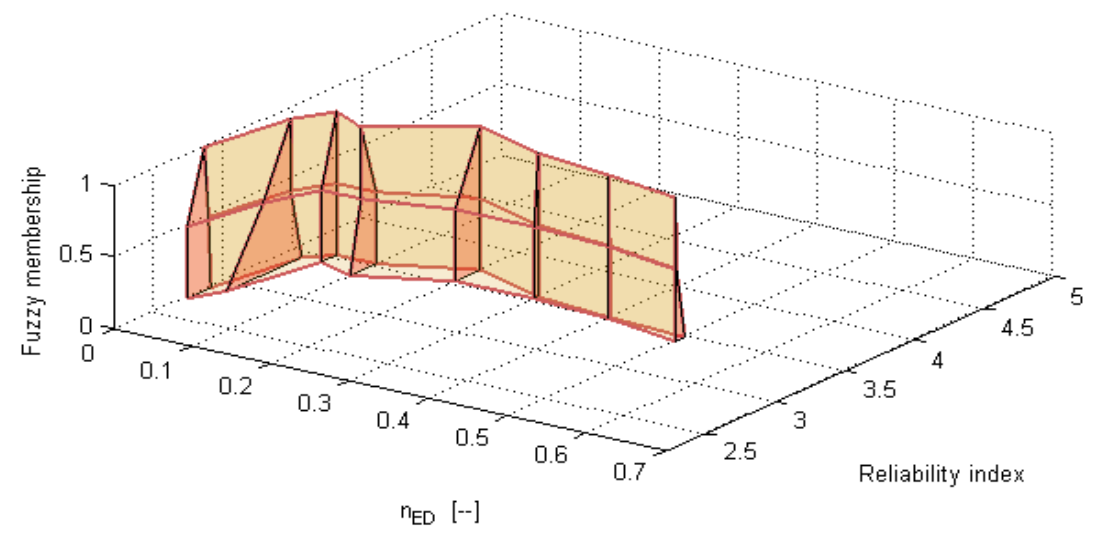

Figure 7. 3D-Reliability of CS URMW $(\psi=1.0)$ through Fuzzy-Random data designed due to DIN EN1996-1-1/NA 


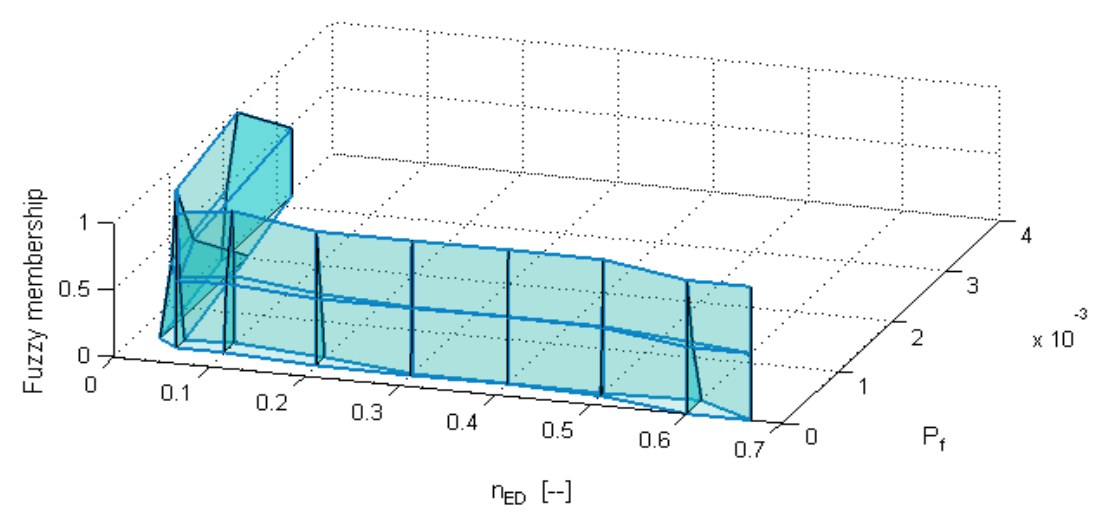

Figure 8. 3D-Failure probability of CS URMW ( $\psi=0.5)$ through Fuzzy-Random data designed due to DIN EN1996-1-1/NA

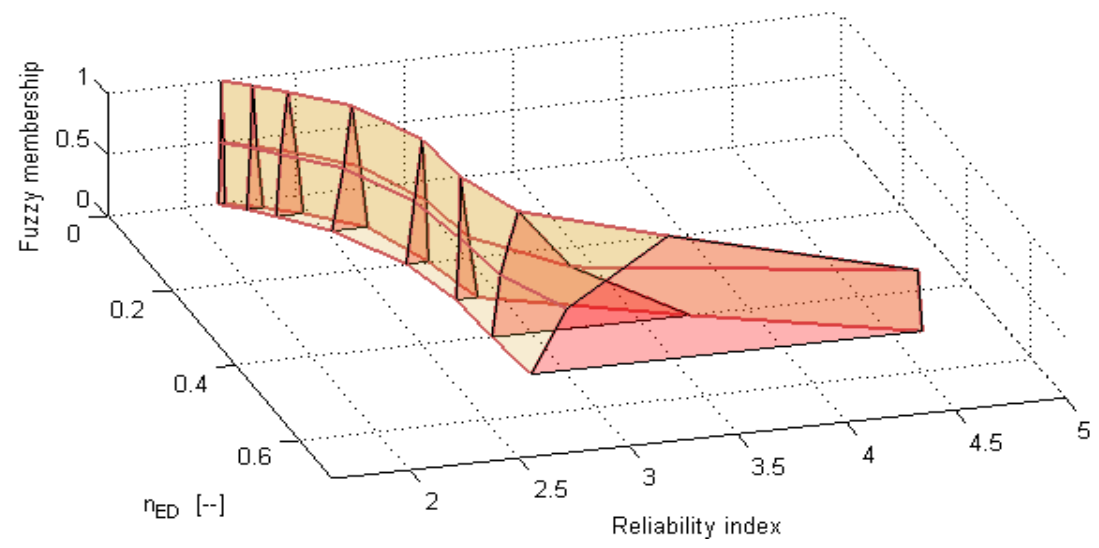

Figure 9. 3D-Reliability of AAC URMW $(\psi=1.0)$ through Fuzzy-Random data designed due to DIN EN1996-1-1/NA

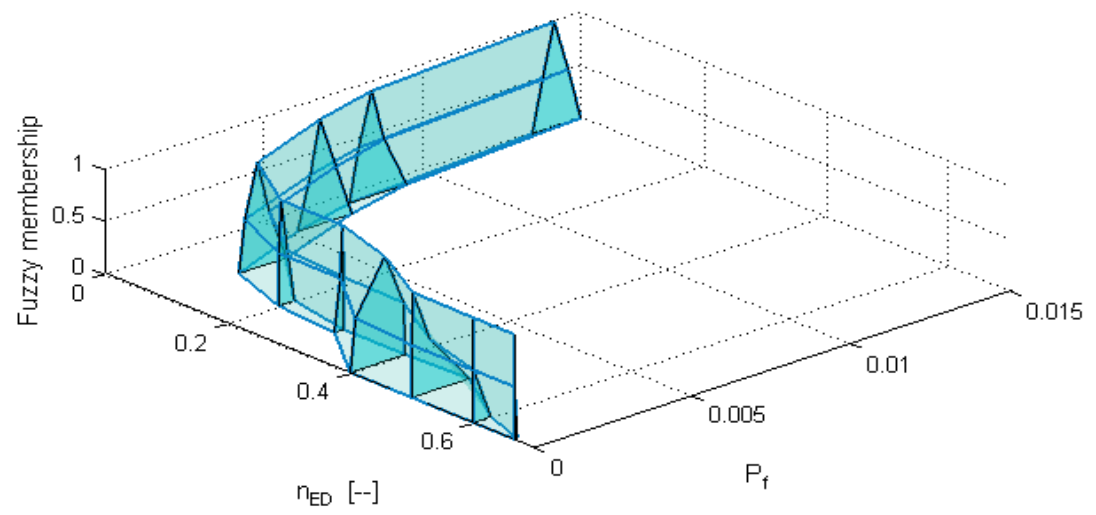

Figure 10. 3D-Failure probability of AAC URMW $(\psi=0.5)$ through Fuzzy-Random data designed due to DIN EN1996-1-1/NA

\section{Conclusion}

The effect of imprecision and incompleteness of data, which are the constituent of epistemic uncertainty, is assessed. It is found that the epistemic uncertainty has major influence on the results (of the reliability level of URMW). The expert opinion should be a part of design to ensure the reliable outcome. It is observed that fuzzy 
set is a very powerful and appropriate means in implementing the epistemic uncertainty, even though it demands numerical efforts.

Since the uncertainty in engineering problems is related to both epistemic and aleatory components, therefore a combination of fuzzy and randomness for specific analysis like safety and reliability assessment is recommended.

The actual reliability of different unreinforced masonry walls, where all kind of uncertainty and variation in parameters is considered, is assessed according to current German national Annex of EC6. The assessed reliabilities in form of fuzzy numbers provide more insight into behavior of URMW. The founding in the current research may be taken into consideration by code writers to promote the design process described in the codes of practice.

\section{References}

Bandemer, H., \& Gottwald, S. (1989). Einführung in FUZZY-Methoden / Theorie und Anwendungen unscharfer Mengen. Berlin: Akad. Verl.

Beer, M., Tong, Q. S., Mingqiang, Z., \& Ferson, S. (2011). Structural reliability assessment with fuzzy probabilities. Paper presented at the 7th International Symposium on Imprecise Probability: Theories and Applications, Innsbruck, Austria. http://www.sipta.org/isipta11/proceedings/papers/s003.pdf

Blake, R. E. (1966). On predicting structural reliability. Paper presented at the 4th Aerospace Sciences Meeting, Fourth Annual Reliability and Maintainability Conference, AIAA paper, California. http://arc.aiaa.org/doi/pdf/10.2514/6.1966-503

Cai, K. Y. (1996). Introduction to fuzzy reliability. Boston: Kluwer.

Chachi, J., \& Taheri, S. M. (2011). Fuzzy confidence intervals for mean of Gaussian fuzzy random variables. Expert Systems with Applications, 38(5), 5240-5244. http://dx.doi.org/10.1016/j.eswa.2010.10.034

DIN. (2012). Nationaler Anhang -National festgelegte Parameter -Eurocode 6 Teil 1-1: Allgemeine Regeln für bewehrtes und unbewehrtes Mauerwerk (pp. 68). Berlin: Deutsches Institut für Normung.

Graf, W., Götz, M., \& Kaliske, M. (2014). Analysis of dynamical processes under consideration of polymorphic uncertainty. Structural Safety, 52, Part B(0), 194-201. http://dx.doi.org/10.1016/j.strusafe.2014.09.003

Grosse, C. U. (2007). Advances in construction materials 2007. Berlin: Springer Science \& Business Media.

Kacprzyk, J., \& Fedrizzi, M. (1988). Combining fuzzy imprecision with probabilistic uncertainty in decision making (Vol. 310). Berlin: Springer.

Kiureghian, A. D., \& Ditlevsen, O. (2009). Aleatory or epistemic? Does it matter? Structural Safety, 31(2), 105-112.

Kranzler, T. (2008). Tragfähigkeit überwiegend horizontal beanspruchter Aussteifungsscheiben aus unbewehrtem Mauerwerk. (PhD), Technische Universität Darmstadt, Germany. Retrieved from http://www.massivbau.tu-darmstadt.de/massivbau/publikationen_fgm/dissertationen_4/index.de.jsp

Mann, W., \& Müller, H. (1978). Schubtragfähigkeit von Mauerwerk. Mauerwerk-Kalender, 3, 35-65.

Möller, B., \& Beer, M. (2004). Fuzzy randomness: uncertainty in civil engineering and computational mechanics (Edition ed.). Berlin: Springer.

Möller, B., Beer, M., \& Liebscher, M. (2005). Fuzzy Analysis as Alternative to Stochastic Methods: Theoretical Aspects. Paper presented at the 4th German LS-DYNA Forum, Bamberg. URL

Möller, B., Graf, W., \& Beer, M. (2000). Fuzzy structural analysis using $\alpha$-level optimization. Computational Mechanics, 26(6), 547-565. http://10.1007/s004660000204

Möller, B., Graf, W., \& Beer, M. (2000). Fuzzy-Tragwerksanalyse - Tragwerksanalyse mit unscharfen Parametern. [Fuzzy Structural Analysis - Structural analysis with uncertain parameters]. Bauingenieur, 75(11), 697-708.

Möller, B., Graf, W., \& Beer, M. (2003). Safety assessment of structures in view of fuzzy randomness. $\begin{array}{lllll}\text { [TranslatedTitle]. } & \text { Computers } \& \quad \text { Structures, } & 81(15), & 1567-1582 .\end{array}$ http://dx.doi.org/10.1016/S0045-7949(03)00147-0

Möller, B., Graf, W., \& Nguyen, S. H. (2001). Uncertainty in Damage Assessment of Structures and Its Numerical Simulation. Computer-Aided Civil and Infrastructure Engineering, 16(6), 375-383. 
http://dx.doi.org/10.1111/0885-9507.00240

Möller, B., Graf, W., Hoffmann, A., \& Sickert, J. U. (2007). Uncertainty models for safety assessment of textile reinforced concrete structures. In C. U. Grosse (Ed.), Advances in Construction Materials 2007 (Vol. 1, pp. 67-74): Springer Berlin Heidelberg.

Möller, B., Graf, W., Sickert, J. U., \& Reuter, U. (2007). Numerical simulation based on fuzzy stochastic analysis. [TranslatedTitle]. Mathematical and Computer Modelling of Dynamical Systems, 13(4), 349-364.

Montazerolghaem, M., Sickert, J. U., Graf, W., \& Jäger, W. (2011). Fuzzy and Reliability Analysis of Unreinforced Masonry Walls subjected to In-Plane Shear Loading. Paper presented at the 9th International Probabilistic Workshop, Braunschweig, Germany. Retrieved from http://forschungsinfo.tu-dresden.de/detail/publikation/a38783

Pannier, S., Waurick, M., Graf, W., \& Kaliske, M. (2013). Solutions to problems with imprecise data-An engineering perspective to generalized uncertainty models. Mechanical Systems and Signal Processing, 37(1-2), 105-120. http://dx.doi.org/10.1016/j.ymssp.2012.08.002

Reuter, U. (2009). Modellierung ungewisser Daten des Bauingenieurwesens und Anwendung der Modelle in Statik und Dynamik. Dresden: Techn. Univ., Inst. für Statik und Dynamik der Tragwerke.

Simon, E. (2002). Schubtragverhalten von Mauerwerk aus großformatigen Steinen. (PhD), Technische Universität Darmstadt, Darmstadt, Germany. Retrieved from http://www.massivbau.tu-darmstadt.de/massivbau/publikationen_fgm/dissertationen_4/index.de.jsp

Viertl, R. (1996). Statistical methods for non-precise data (Edition ed.): CRC Press.

Viertl, R. (1997). On Statistical Inference for Non-Precise Data. [TranslatedTitle]. Environmetrics, 8(5), 541-568. http://dx.doi.org/10.1002/(SICI)1099-095X(199709/10)8:5<541::AID-ENV269>3.0.CO;2-U

Yubin, L., Zhong, Q., \& Guangyuan, W. (1997). Fuzzy random reliability of structures based on fuzzy random variables. Fuzzy Sets and Systems, 86(3), 345-355. http://dx.doi.org/10.1016/S0165-0114(96)00002-4

Zadeh, L. A. (1965). Fuzzy sets. Information and Control, 8(3), 338-353. http://dx.doi.org/10.1016/S0019-9958(65)90241-X

Zadeh, L. A. (1984). Fuzzy probabilities. Information Processing \& Management, 20(3), 363-372. http://dx.doi.org/10.1016/0306-4573(84)90067-0

Notes

Note 1. http://tu-dresden.de/die_tu_dresden/fakultaeten/fakultaet_bauingenieurwesen/sdt/software/fuzzy/fly.pdf

\section{Copyrights}

Copyright for this article is retained by the author(s), with first publication rights granted to the journal.

This is an open-access article distributed under the terms and conditions of the Creative Commons Attribution license (http://creativecommons.org/licenses/by/3.0/). 